This is the peer reviewed version of the following article:

E. Barrigón, L. Barrutia, M. Ochoa, I. Rey-Stolle, and C. Algora, "Effect of Sb on the quantum efficiency of GalnP solar cells," Progress in Photovoltaics: Research and Applications, 2016.

which has been published in final form at

http://onlinelibrary.wiley.com/wol1/doi/10.1002/pip.2777/abstract

This article may be used for non-commercial purposes in accordance with Wiley Terms and Conditions for Self-Archiving." 


\title{
Effect of Sb on the Quantum Efficiency of GaInP Solar Cells
}

\author{
Enrique Barrigón, Laura Barrutia, Mario Ochoa, Ignacio \\ Rey-Stolle, and Carlos Algora \\ Instituto de Energía Solar, Escuela Técnica Superior de Ingenieros de \\ Telecomunicación, Universidad Politécnica de Madrid, Avda. Complutense 30, 28040 \\ Madrid, Spain
}

\begin{abstract}
$1 \quad$ Abstract
2 The energy bandgap of GaInP solar cells can be tuned by modifying the 3 degree of order of the alloy. In this study, we employed Sb to increase the 4 energy bandgap of the GaInP and analyzed its impact on the performance of 5 GaInP solar cells. An effective change in the cut-off wavelength of the external 6 quantum efficiency of GaInP solar cells and an effective increase of $50 \mathrm{mV}$ in 7 the open circuit voltage of $\mathrm{GaInP} / \mathrm{Ga}(\mathrm{In}) \mathrm{As} / \mathrm{Ge}$ triple junction solar cells were 8 obtained with the use of Sb.
\end{abstract}

\section{${ }_{10} 1$ Introduction}

11 The performance optimization of GaInP subcells is key to obtain multijunction 12 solar cells (MJSC) with high efficiency. To date, all MJSC architectures [1, $132,3,4,5]$ employ GaInP-based subcells, whereas their differences rely on the 14 materials, composition and structure of the other subcells. Nonetheless, the 15 exact composition, energy band gap $\left(E_{g}\right)$ and thickness of the GaInP subcell 16 should be carefully controlled since they directly influence its photocurrent and 17 subsequently that of the underlying subcells. Hence, the current mismatch in 18 a MJSC under a given spectrum can be impacted (even some times controlled) 19 with any of these three magnitudes [6].

20 In particular, GaInP exhibits CuPt-type ordering in the group III sublattice 21 which modifies the $E_{g}$ of the alloy [7, 8]. For a fully disordered GaInP (i.e., 22 with $\mathrm{Ga}$ and In atoms randomly located in the group III lattice sites) $E_{g}$ can ${ }_{23}$ be raised up to $100 \mathrm{meV}$ [9]. In order to raise the efficiency of a GaInP subcell 
in a MJSC, its $E_{g}$ should be increased as much as possible (i.e., GaInP should be disordered) [6], while keeping the photocurrent as high as possible. To this end, typical epitaxial growth parameters such as growth temperature, growth rate, V/III ratio, doping level, wafer off cut $[10,11,12,13]$ can be manipulated. However, a change in the growth parameters may also affect the minority and majority carrier properties, which directly impact the performance of the solar cell. Alternatively, surfactants can be employed during the epitaxial process to modulate the degree of order without changing other process variables [14]. Typical surfactants of GaInP include Sb [15], Te [16] or Bi [17]. To date, only $\mathrm{Sb}$ has been employed to modulate the order parameter in GaInP solar cells and, by doing so, up to $60 \mathrm{mV}$ increase in the $V_{o c}$ was obtained [9]. However, there is no detailed information concerning the impact of the use of Sb during GaInP growth on the external quantum efficiency (EQE) of GaInP solar cells, which in turn determines the photocurrent of the solar cell. In the present study, we show the relative impact of the ordering of the base and the emitter of the GaInP solar cell on the shape of its EQE, together with the global effect of GaInP ordering on the EQE of a typical MJSC. We also carried out simulations to confirm the experimental observations.

\section{Experimental}

Solar cells were grown in a commercial Aixtron 200/4 MOVPE reactor on ptype $\mathrm{Ge}(100)$ wafers with $6^{\circ}$ misorientation towards [011] . The semiconductor structure, together with the thickness and the dopant value of each layer, can be seen in Fig.1. A double GaInP/GaInAs nucleation layer was employed [18] to create a high quality template for the subsequent growth of a tunnel junction [19] and the GaInP solar cell. The tunnel junction was introduced since a diffused emitter is created during the growth of the nucleation layer, forming a $p-n$ junction in the Ge substrate. The details behind the semiconductor structure of the GaInP solar cell can be found in Ref. [20]. The Sb molar flow used during the growth is expressed in terms of the $\mathrm{Sb} / \mathrm{P}$ ratio in the gas phase, in parts per million (ppm), under a constant $\mathrm{PH}_{3}$ molar flow of $1.79 \cdot 10^{-2} \mathrm{~mol} / \mathrm{min}$. The effect of $\mathrm{Sb}$ is very sensitive to growth conditions and reactor geometry so we have developed a method to calibrate the injected molar flow of TESb to obtain a desired order parameter based on Reflectance Anisotropy Spectroscopy (RAS) [21]. Sb was mainly employed during the growth of the base layer, as the emitter showed a low order parameter as a result of its high doping level [22]. As it will be explained later, the molar flow of the dopant in the base layer (DMZn) was slightly modified in each sample to counterbalance the impact of $\mathrm{Sb}$ that enhances the incorporation of $\mathrm{Zn}$ into the solid [22, 23]. 
The setup employed for the EQE measurements consists of a Xe lamp used as white light source which passes through a Horiba Jobin Yvon monochromator (TRIAX180) and a filter wheel. Further details on the system and the measurement can be found in [24]. Measurements under concentration were done with a flash simulator [25]. Solar cells were also modelled with a 2-D physically-based numerical modeling tool (Atlas from Silvaco). This type of modeling solves numerically the fundamental semiconductor equations under specified bias conditions [26]. Thermionic and thermionic field emission boundary conditions were used to model the non-linear transport at heterojunctions. The transfer matrix method was used to calculate the photo generation rate through the structure which accurately takes into account constructive and/or destructive interferences at the interfaces of the solar cell [27]. More details about the modeling approach can be found elsewhere [28].

\section{Theoretical assessment}

The $V_{o c}$ of GaInP solar cells is enhanced by increasing its $E_{g}$ with the use of $\mathrm{Sb}$ to disorder the GaInP [9]. However, the photocurrent gets reduced by increasing the band gap due to the lower cut-off wavelength in the absorption coefficient of the material. Therefore, to increase the efficiency of the solar cell one must raise the $V_{o c}$ while keeping the photocurrent as high as possible. To preserve the photocurrent, the top cell thickness base is typically used as an adjustable parameter to achieve current-matching in a MJSC device [6]. However, a base thickness increase leads to a $V_{o c}$ decrease. Accordingly, Fig.2 illustrates the solution of this trade-off for solar cell architectures in which GaInP lattice matched to Ge (or GaAs) is used as the top cell (for example, state-of-the-art GaInP/Ga(In)As/Ge solar cells; inverted metamorphic GaInP/Ga(In)As/GaInAs solar cells or GaInP/Ga(In)As/GaInAsN solar cells). In particular, Fig.2 depicts the evolution of $J_{s c}$ and $V_{o c}$ as a function of the thickness and $E_{g}$ of the base layer in the GaInP subcell for a typical GaInP/Ga(In)As/Ge MJSC under AM1.5D ASTM G173-03 spectrum. The bandgap range considered is something attainable by controlling the ordering around the GaInP composition considered in the example. For the simulation, it has been considered that 1 ) the photogeneration of carriers is zero for photons with energies below $E_{g}\left(\mathrm{k}=0\right.$ for $E_{p h}<E_{g}$ and 2) there is no degradation of the minority carrier properties due to the use of Sb. As can be seen in Fig.2(a), the $J_{s c}$ is very sensitive to the base thickness, especially for low $E_{g}{ }^{\prime} \mathrm{s}$. A $J_{s c}$ gain of around $3 \mathrm{~mA} / \mathrm{cm}^{2}$ can be obtained by increasing the base thickness from 300 to $1600 \mathrm{~nm}$ for low band gaps (1.8-1.84 eV). For mid-high band gaps, a lower increase is expected (about 1.5 to $2 \mathrm{~mA} / \mathrm{cm}^{2}$ ). Regarding the $V_{o c}$ (see 
Fig.2(b)), the highest values are expected for high $E_{g}$ and relatively thin base thicknesses (300-700 nm). For example, for a base thickness of $500 \mathrm{~nm}$ a gain of $50 \mathrm{mV}$ is feasible by increasing $E_{g}$ from 1810 to $1880 \mathrm{meV}$. However, as the base thickness must be as high as possible to keep the same current levels when using high bandgaps, the dark saturation current is also increased and consequently the $V_{o c}$ is reduced. Nonetheless, the net change in $V_{o c}$ due to the increase of $E_{g}$ and base thickness is positive, which justifies the procedure. Besides, the dark saturation current impact on the $V_{o c}$ gets reduced as the band gap is increased (almost negligible for $E_{g}>1.88 \mathrm{eV}$ ) due to a reduction of the intrinsic carrier concentration [29]. Summarizing, according to the simulations it is reasonable to go for higher band gaps to raise the $V_{o c}$ and to use the base thickness to adjust the photocurrent in any particular multijunction solar cell design.

\section{Results and discussion}

Fig. 3 shows the EQE of several GaInP subcells with the semiconductor structure of Fig. 1, that were grown with different $\mathrm{Sb} / \mathrm{P}$ ratios as shown in the legend. Despite the solar cells in Fig. 1 are GaInP/Ge dual junction solar cells, we will ignore in this work the EQE of the Ge cell since it does not have any relevance for our study. No antireflection coating (ARC) was deposited on the devices. The EQE represented with black squares corresponds to the reference solar cell with no Sb flow during its growth. As can be observed, when higher $\mathrm{Sb} / \mathrm{P}$ ratios were employed during the GaInP solar cell base growth, the cut-off wavelength in the EQE was shifted to lower wavelengths (i.e., higher energies) indicating an effective energy bandgap increase. Olson et al. [9] reported that $\mathrm{Sb}$ modifies the alloy composition (i.e. Ga to In ratio) depending on the substrate offcut. Nonetheless, we have experimentally observed with XRD measurements a change from 0.502 to 0.507 in Ga composition, which yields a negligible change in the $E_{g}$.

On the other hand, samples with $\mathrm{Sb} / \mathrm{P}$ ratios of 1720 and $2610 \mathrm{ppm}$ showed an EQE markedly lower than the rest of the samples. The reason for this is that the use of Sb during Zn-doped GaInP layer growth increases dopant incorporation $[22,23]$. As a result, the minority carrier lifetime of electrons in the base was lowered and correspondingly the EQE decreased. Therefore, the change in the EQE observed is related to the change in the dopant incorporation which has not been correctly compensated by reducing the dopant flow. Nonetheless, the ultimate goal here was to firstly address the shift in the cut-off wavelength due to the effect of the use of Sb. Later on, it will be shown in Fig.4 that the correction of the Zn concentration in the base leads to the obtaining of an EQE in the samples grown using Sb that virtually overlaps that of the reference cell 
except for the change in the cut-off wavelength region.

Fig. 3(b) shows the cut-off wavelength region magnified to facilitate the analysis. As can be observed, a little hump appears at high wavelengths (identified in the figure with a red rectangle), as the $E_{g}$ is increased. The relative influence of this hump in the integrated photocurrent was higher as the cut-off wavelength was decreased. In fact, for the sample with the highest $\mathrm{Sb} / \mathrm{P}$ ratio (i.e., highest $E_{g}$ ), a current "gain" of $0.15 \mathrm{~mA} / \mathrm{cm}^{2}$ can be estimated as a result of this hump. The presence of such hump may be indicative of areas within the structure with a higher degree of order (i.e., lower $E_{g}$ ) than in the base layer. As Sb flow was only introduced during the growth of the base layer, the hump in the EQE could be the result of the emitter having a higher degree of order than the base layers grown with high $\mathrm{Sb} / \mathrm{P}$ ratios. In order to shed some light on this issue, Fig. 3(b) also shows the simulation performed of GaInP solar cells (dashed lines) considering a more ordered emitter than the highly disordered base. Indeed, as Fig. 3(b) shows, the measurements can be fitted quite closely considering the $E_{g}$ of the emitter to be $25 \mathrm{meV}$ lower than the base (2610 ppm case).

Accordingly, in order to get rid of the hump in the EQE (i.e. to get a fully disordered emitter), an Sb flow needs to be also introduced during the growth of the emitter layer. Fig.4 shows the EQEs of such batch of experiments. Again, the EQE of the reference structure grown with no $\mathrm{Sb}$ is represented with black squares while now the EQE of a GaInP solar cell grown with an $\mathrm{Sb} / \mathrm{P}$ ratio of $2610 \mathrm{ppm}$ during the base growth is shown with red circles. This solar cell was similar to the solar cell whose EQE was plotted with light blue diamonds in Fig.3. Curves plotted with green and blue triangles correspond to solar cells grown with an $\mathrm{Sb} / \mathrm{P}$ ratio of 728 and $2610 \mathrm{ppm}$ in the emitter, respectively, and an $\mathrm{Sb} / \mathrm{P}$ ratio of $2610 \mathrm{ppm}$ in the base. As can be observed, as the amount of $\mathrm{Sb}$ introduced during the growth of the emitter increases, the hump in the cut-off wavelength region tends to vanish. This was indicative that the emitter was decreasing its degree of order, attaining values at least similar to that of the base. Furthermore, the solar cells grown with an $\mathrm{Sb} / \mathrm{P}$ ratio of $728 \mathrm{ppm}$ in the emitter (green line of Fig. 4) and without Sb (red line of Fig. 4) in the emitter were grown virtually identical with the exception of the use of $\mathrm{Sb}$ in the emitter. Since for these cells the EQEs are coincident for all wavelengths (excluding the hump region), it can be concluded that there were apparently no major issues concerning the n-type dopant (i.e., Si) incorporation in the emitter when using such Sb quantity. Finally, for the sample grown with an Sb/P ratio of 2610 ppm along both the base and emitter (blue line in Fig. 4), the Zn-molar flow in the base was corrected to compensate the enhanced $\mathrm{Zn}$ incorporation. Fig. 4 shows that the result of such compensation is a recovery of the EQE, virtually 
showing the same EQE values as the reference solar cell grown with no Sb flow. Although it has been reported that the use of Sb reduces the minority carrier lifetime [30], we have found it does not affect the EQE of practical devices for the range of $E_{g}$ we have explored.

The cut-off wavelength shift of the GaInP towards higher energies has direct consequences in the performance of the GaInP solar cell when it is employed as a top cell in a MJSC. The shift in the cut-off wavelength not only implies an increase in $V_{o c}$ but also a decrease in $J_{s c}$ since there is a significant loss in absorption in the range from 610 to $700 \mathrm{~nm}$. For instance, a $J_{s c}$ loss of $1.16 \mathrm{~mA} / \mathrm{cm}^{2}$ is calculated for the AM1.5D ASTM G173-03 spectrum in such region for the sample grown with an $\mathrm{Sb} / \mathrm{P}$ ratio of $2610 \mathrm{ppm}$ in the base and the emitter of Fig.4 (with respect to the reference). As seen in the theoretical assessment section, in order to counterbalance such $J_{s c}$ decrease, the top cell base thickness could be increased. To illustrate this issue, two different latticematched $\mathrm{GaInP} / \mathrm{Ga}(\mathrm{In}) \mathrm{As} / \mathrm{Ge}$ triple junction solar cells (3JSC) were grown and their EQEs can be seen in Fig.5. The EQE with black squares corresponds to a reference sample grown with no $\mathrm{Sb}$ in the TC (i.e., ordered GaInP TC), while the EQE with red circles corresponds to a 3JSC with Sb only in the base of the TC (i.e., disordered GaInP TC). Details on the growth and manufacturing can be found in Ref. [31]. The EQE of the GaInP top cells (TC) and Ga(In)As middle cells (MC) range from 300 to $700 \mathrm{~nm}$ and 500 to $950 \mathrm{~nm}$, respectively. The EQE of the Ge bottom cell is not included in the figure since it does not experience any change after the alteration of the $E_{g}$ of the TC. The shape of the EQE of the GaInP subcells shows several differences. First, as expected, the cut-off wavelength has been decreased after using Sb during the growth. Second, in the case of the cell using $\mathrm{Sb}$, the EQE has been increased in the range of 550 to $650 \mathrm{~nm}$ as a consequence of the increase of the GaInP base thickness (from 600 to $1300 \mathrm{~nm}$ in this case) implemented to counterbalance the photocurrent loss due to decrease of the cut-off wavelength. However, as it will be shown later, despite the increase in base thickness the disordered TC could not reach the equivalent $J_{s c}$ level of the ordered TC. This impacted the current matching in the disordered 3JSC. Finally, it should be noticed that the differences in the EQE of Fig.5 in the short wavelength region (from 300-350 nm) are attributed to slight differences in the surface recombination velocity of the AlInP window layer between both solar cells, and have nothing to do with the use of Sb.

Regarding the EQE of the $\mathrm{Ga}(\mathrm{In}) \mathrm{As} \mathrm{MC}$, it was also affected by the changes introduced. On the one hand, it could be argued that, as the TC is absorbing less light (higher $E_{g}$ ), the MC should increase its EQE in the range from 500 to $700 \mathrm{~nm}$. However, this is not the case since the base thickness increase in the TC changes the expected behavior. The EQE between 650 and $700 \mathrm{~nm}$ 
increases, due to the shift in the cut-off wavelength of the TC, whereas the EQE between 500 and $650 \mathrm{~nm}$ decreases, due to the TC-base thickness increase. Fig.5 (b) shows the same EQEs as in (a) but with an enlarged X-axis between 550 and $700 \mathrm{~nm}$. In the end, the current gain obtained in the MC thanks to the change in $E_{g}$ of the TC virtually counterbalances the current loss due to the increased TC-base thickness for a given reference spectrum. In other words, the photocurrent produced by both middle cells with (red curve in Fig. 5) and without $\mathrm{Sb}$ (black curve in Fig. 5) is almost the same $\left(0.05 \mathrm{~mA} / \mathrm{cm}^{2}\right.$ current gain in the MC after using $\mathrm{Sb}$ ). As a rule of thumb, in order to optimize the efficiency of the triple junction solar cell with regard to the $E_{g}$ and thickness of the TC, the first step is to achieve a TC with the highest energy bandgap possible since the higher the energy bandgap, the higher the $V_{o c}$ of the device. Afterwards, the current matching between the TC and the MC under a given spectrum is obtained by fine tuning the TC-thickness.

Finally, in order to quantify the increase in $V_{o c}$ due to the use of $\mathrm{Sb}$ to get a disordered GaInP subcell, the two triple junction solar cells of Fig. 5 were measured at an equivalent concentration of 500 suns (see Fig.6). With the use of $\mathrm{Sb}$ in the TC base, an increase in $V_{o c}$ of $51 \mathrm{mV}$ has been attained, due to the change in the $E_{g}$ of the TC. Although it might be a low value in comparison to values published in the literature [9], the result seems reasonable after considering the decrease in the $V_{o c}$ due to the thickness base increase. Indeed, after the simulations from Fig.2, an $V_{o c}$ loss of $10 \mathrm{mV}$ can be expected after an increase of the base layer from 600 to $1300 \mathrm{~nm}$. On the other hand, as mentioned above, the slight decrease in current (an equivalent loss of $0.23 \mathrm{~mA} / \mathrm{cm}^{2}$ at $1 \mathrm{sun}$ ) is due to a slight current mismatch between the TC and $\mathrm{MC}$ originated by the not fully optimized EQE of the TC (see for instance the room for improvement at $500 \mathrm{~nm}$ ). The current mismatch between the subcells could be also solved with a careful design of an ARC layer (devices in this work have no ARC layer). Nonetheless, we want to stress here the efficiency potential gain in the 3JSC ( $0.7 \%$ absolute according to our calculations) due to the $V_{o c}$ increase once the current matching issues are correctly solved. Alternatively, in some other MJSC architectures there may be an interest in decreasing the current of the GaInP subcell. In such case, the use of Sb could be employed to both decrease the current and increase the $V_{o c}$ at the same time.

\section{Conclusions}

In summary, an effective decrease in the cutoff wavelength in the EQE of GaInP solar cells (i.e., an increase in the energy bandgap) has been obtained with the use of $\mathrm{Sb}$ during the growth of the GaInP material. In addition, the particular 
degree of order of the solar cell emitter and base layers influences the shape of the EQE of the devices and the use of Sb does not seem to affect the minority carrier lifetime of the solar cells tested. Once incorporated in a MJSC architecture, the thickness of the GaInP base must be increased to counterbalance the current loss originated by the change in the energy bandgap. Finally, an increase of $50 \mathrm{mV}$ has been also obtained with the use of $\mathrm{Sb}$ in state-of-the-art $\mathrm{GaInP} / \mathrm{Ga}(\mathrm{In}) \mathrm{As} / \mathrm{Ge}$ triple junction solar cells.

\section{Acknowledgments}

This work has been supported by the Spanish MINECO through the projects TEC2012-37286, TEC2014-54260-C3-1-P and RTC-2015-3747-3, and by the Comunidad de Madrid through the project MADRID-PV (S2013/MAE-2780). The authors would also like to thank Jesus Bautista for his continuous support.

\section{References}

[1] F. Dimroth, M. Grave, P. Beutel, U. Fiedeler, C. Karcher, T. Tibbits, E. Oliva, G. Siefer, M. Schachtner, A. Wekkeli, A. W. Bett, R. Krause, M. Piccin, N. Blanc, C. Drazek, E. Guiot, B. Ghyselen, T. Salvetat, A. Tauzin, T. Signamarcheix, A. Dobrich, T. Hannappel, and K. Schwarzburg, "Wafer bonded four-junction GaInP/GaAs//GaInAsP/GaInAs concentrator solar cells with $44.7 \%$ efficiency," Progress in Photovoltaics: Research and Applications, vol. 22, no. 3, pp. 277-282, 2014.

[2] V. Sabnis, H. Yuen, and M. Wiemer, "High-efficiency multijunction solar cells employing dilute nitrides," AIP Conf. Proc., vol. 1477, no. 1, pp. 14-19, 2012.

[3] A. Yoshida, T. Agui, N. Katsuya, K. Murasawa, H. Juso, K. Sasaki, and T. Takamoto, "Development of InGaP/GaAs/InGaAs inverted triple junction solar cells for concentrator application," in 21st International Photovoltaic Science and Engineering Conference (PVSEC-21), 2011.

[4] J. F. Geisz, D. J. Friedman, J. S. Ward, A. Duda, W. J. Olavarria, T. E. Moriarty, J. T. Kiehl, M. J. Romero, A. G. Norman, , and K. M. Jones, " $40.8 \%$ efficient inverted triple-junction solar cell with two independently metamorphic junctions," Appl. Phys. Lett., vol. 93 123505-1, 2008.

[5] R. R. King, D. C. Law, K. M. Edmondson, C. M. Fetzer, G. S. Kinsey, H. Yoon, R. A. Sherif, and N. H. Karam, " $40 \%$ efficient metamorphic 
GaInP/GaInAs/Ge multijunction solar cells," Appl. Phys. Lett., vol. 90, no. 18, APR 302007.

[6] Sarah R. Kurtz, P. Faine, and J. M. Olson, "Modeling of two-junction, series-connected tandem solar cells using top-cell thickness as an adjustable parameter.," J. Appl. Phys., vol. 68, no. 4, pp. 1890, 1990.

[7] A. Gomyo, T. Suzuki, and S. Iijima, "Observation of Strong Ordering in GaInP alloy semiconductors," Phys. Rev. Lett., vol. 60, pp. 2645-2648, Jun 1988.

[8] A. Gomyo, T. Suzuki, K. Kobayashi, S. Kawata, I. Hino, and T. Yuasa, "Evidence for the existence of an ordered state in $\mathrm{Ga}_{0.5} \operatorname{In}_{0.5} \mathrm{P}$ grown by metalorganic vapor phase epitaxy and its relation to band-gap energy.," Appl. Phys. Lett., vol. 50, no. 11, pp. 673, 1987.

[9] J.M. Olson, W.E. McMahon, and Sarah Kurtz, "Effect of Sb on the Properties of GaInP Top Cells," in Conference Record of the 2006 IEEE 4th World Conference on Photovoltaic Energy Conversion,, May 2006, vol. 1, pp. $787-790$.

[10] H. Murata, I. H. Ho, Y. Hosokawa, and G. B. Stringfellow, "Surface photoabsorption study of the effect of substrate misorientation on ordering in GaInP," Appl. Phys. Lett., vol. 68, no. 16, pp. 2237-2239, 1996.

[11] H. Murata, I. H. Ho, L. C. Su, Y. Hosokawa, and G. B. Stringfellow, "Surface photoabsorption study of the effects of growth temperature and V/III ratio on ordering in GaInP," J. Appl. Phys., vol. 79, no. 9, pp. 6895, 1996.

[12] H. Murata, T.C. Hsu, I.H. Ho, L.C. Su, Y. Hosokawa, and G.B. Stringfellow, "Surface photoabsorption study of the effect of V/III ratio on ordering in GaInP," Appl. Phys. Lett., vol. 68, no. 13, pp. 1796, 1996.

[13] Sarah R. Kurtz, J. M. Olson, and A. Kibbler, "Effect of growth rate on the band gap of Ga0.5In0.5P.," Appl. Phys. Lett., vol. 57, no. 18, pp. 1922, 1990.

[14] G.B Stringfellow, J.K Shurtleff, R.T Lee, C.M Fetzer, and S.W Jun, "Surface processes in OMVPE the frontiers," J. Cryst. Growth, vol. 221, pp. 1 $-11,2000$.

[15] J. K. Shurtleff, R. T. Lee, C. M. Fetzer, and G. B. Stringfellow, "Band-gap control of GaInP using Sb as a surfactant," Appl. Phys. Lett., vol. 75, no. 13, pp. 1914-1916, 1999. 
[16] S.H. Lee and C.Y. Fetzer, "Te doping of GaInP: Ordering and step structure.," J. Appl. Phys., vol. 85, no. 7, pp. 3590, 1999.

[17] S.W. Jun, R.T. Lee, C.M. Fetzer, J.K. Shurtleff, G.B. Stringfellow, C.J. Choi, and T.-Y. Seong, "Bi surfactant control of ordering and surface structure in GalnP grown by organometallic vapor phase epitaxy.," $J$. Appl. Phys., vol. 88, no. 7, pp. 4429, 2000.

[18] E. Barrigón, B. Galiana, and I. Rey-Stolle, "Reflectance anisotropy spectroscopy assessment of the MOVPE nucleation of GaInP on germanium (100)," J. Cryst. Growth, vol. 315, no. 1, SI, pp. 22-27, JAN 152011.

[19] I. García, I. Rey-Stolle, and C. Algora, "Performance analysis of AlGaAs/GaAs tunnel junctions for ultra-high concentration photovoltaics," J. Phys. D: Appl. Phys., vol. 45, no. 4, pp. 045101, 2012.

[20] I. García, I. Rey-Stolle, B. Galiana, and C. Algora, "A $32.6 \%$ efficient lattice-matched dual-junction solar cell working at 1000 suns," Appl. Phys. Lett., vol. 94, no. 5, pp. 053509, 2009.

[21] E. Barrigón, L. Barrutia, and I. Rey-Stolle, "Optical in situ calibration of Sb for growing disordered GaInP by MOVPE," Journal of Crystal Growth, vol. 426 , pp. $71-74,2015$.

[22] L. Barrutia, E. Barrigon, L. Lpez-Conesa, J. Rebled, S. Estrad, F. Peir, I. Rey-Stolle, and C. Algora, "On the use of Sb to improve the performance of GaInP subcells of Multijunction Solar Cells," in Photovoltaic Specialist Conference (PVSC), 2015 IEEE 41th, June 2015.

[23] D.C. Chapman, A.D. Howard, and G.B. Stringfellow, "Zn enhancement during surfactant-mediated growth of GaInP and GaP," J. Cryst. Growth, vol. 287 , no. 2 , pp. $647-651,2006$.

[24] E. Barrigon, P. Espinet-Gonzalez, Y. Contreras, and I. Rey-Stolle, "Implications of low breakdown voltage of component subcells on external quantum efficiency measurements of multijunction solar cells," Progress in Photovoltaics: Research and Applications, pp. n/a-n/a, 2015.

[25] C. Dominguez, I. Anton, and G. Sala, "Multijunction solar cell model for translating I-V characteristics as a function of irradiance, spectrum, and cell temperature," Progress in Photovoltaics: Research and Applications, vol. 18, no. 4, pp. 272-284, 2010.

[26] Silvaco International, Atlas user's manual Device Simulation Software, 2015. 
[27] E. Centurioni, "Generalized matrix method for calculation of internal light energy flux in mixed coherent and incoherent multilayers," Appl. Opt., vol. 44, no. 35, pp. 7532-7539, 2005.

[28] M. Baudrit, C. Algora, I. Rey-Stolle, and B. Galiana, "Numerical analysis of GaInP solar cells: Toward advanced photovoltaic devices modeling," in Proceedings of the 5th International Conference on Numerical Simulation of Optoelectronic Devices. IEEE, 2005, pp. 41-42.

[29] Simon M. Sze and Kwok K. Ng, Physics of semiconductor devices, John Wiley \& Sons, 2006.

[30] C. M. Fetzer, R. T. Lee, G. B. Stringfellow, X. Q. Liu, A. Sasaki, and N. Ohno, "Effect of surfactant Sb on carrier lifetime in GaInP epilayers," J. Appl. Phys., vol. 91, no. 1, pp. 199, 2002.

[31] E. Barrigón, I. Rey-Stolle, B. Galiana, I. Garcia, and C. Algora, "GaInP/GaInAs/Ge triple junction solar cells for ultra high concentration," in Proc. Spanish Conference on Electron Devices CDE 2009, 11-13 Feb. 2009, pp. 383-386. 


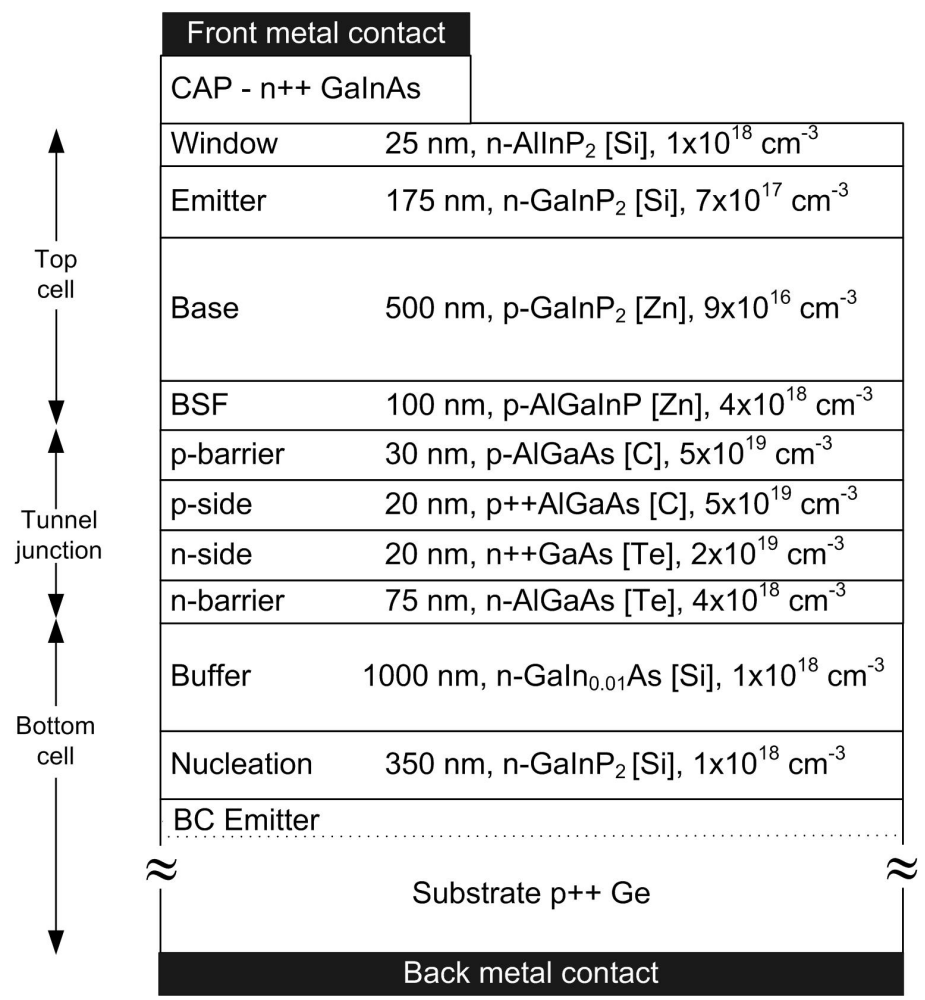

Figure 1: Sketch of the semiconductor structure of the GaInP solar cells analyzed.

(a)

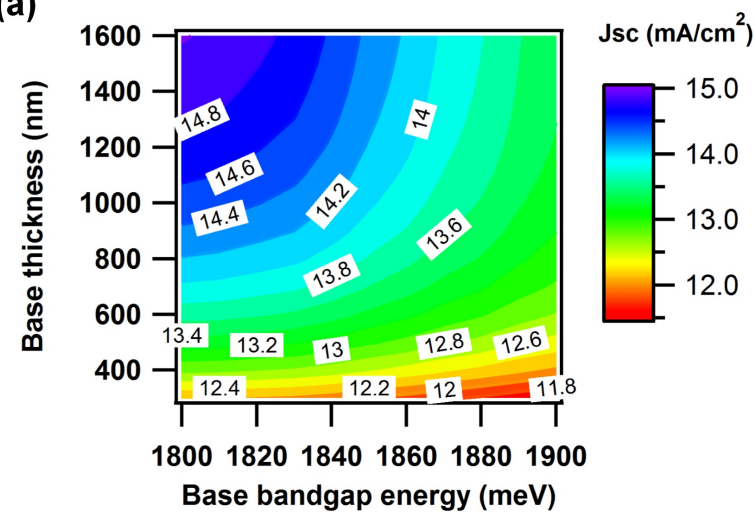

(b)

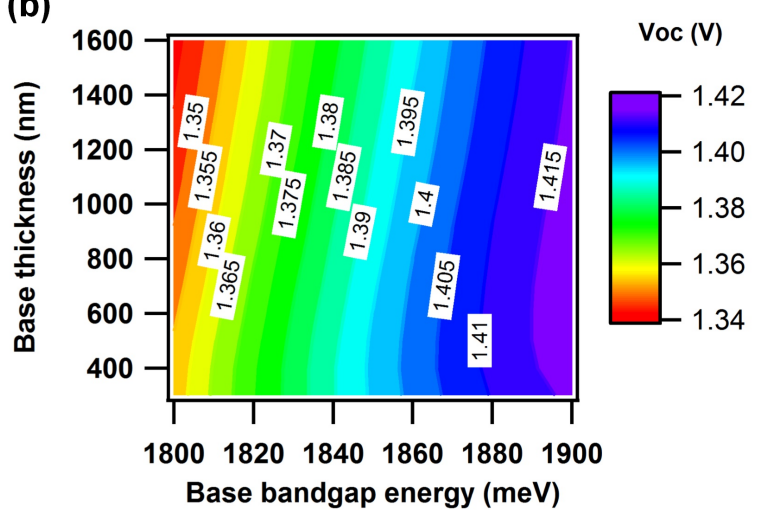

Figure 2: (a) Calculated short circuit current density and (b) open circuit voltage of the GaInP subcell latticed-matched to Ge as a function of the bandgap energy and the thickness of the base layer under AM1.5D ASTM G173-03. 

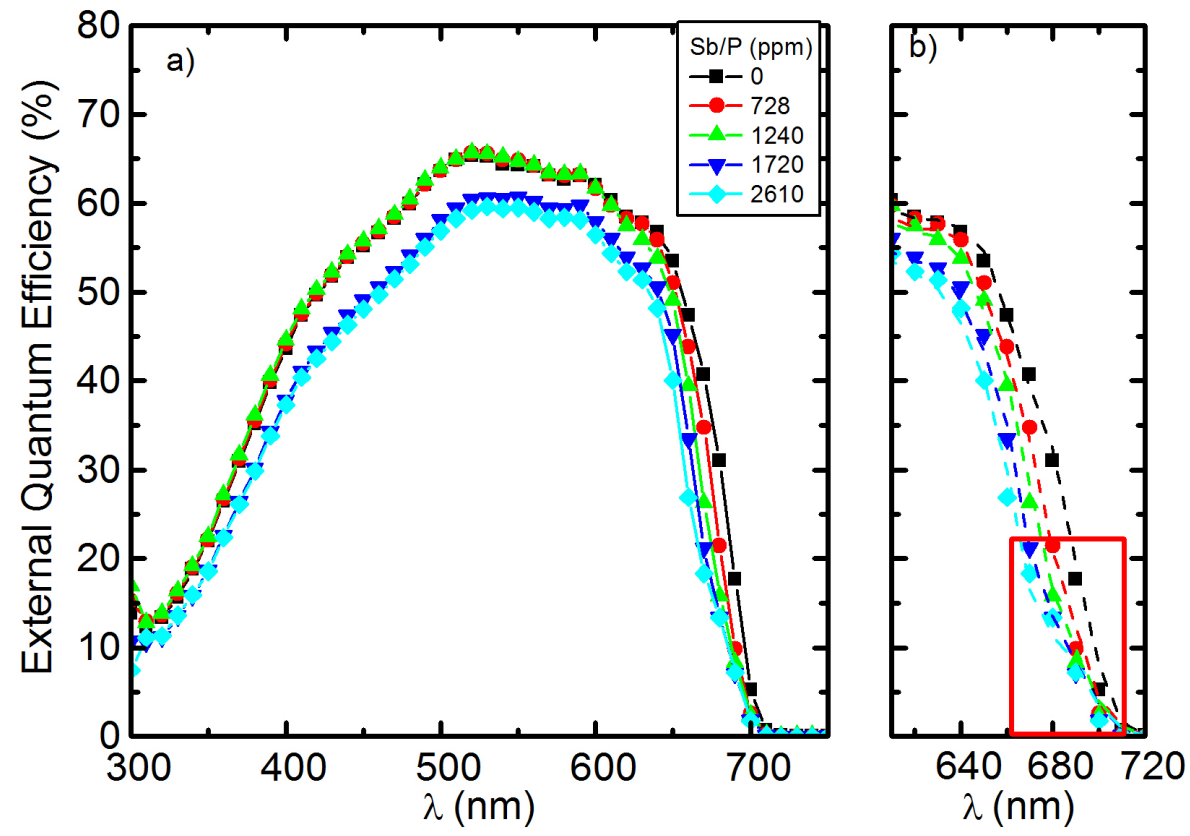

Figure 3: (a) EQE of GaInP solar cells with different $\mathrm{Sb} / \mathrm{P}$ ratios employed during the growth of the base layer. (b) Zoom in of the cut-off wavelength region of plot (a). Dashed lines in this case correspond to the simulations performed. 

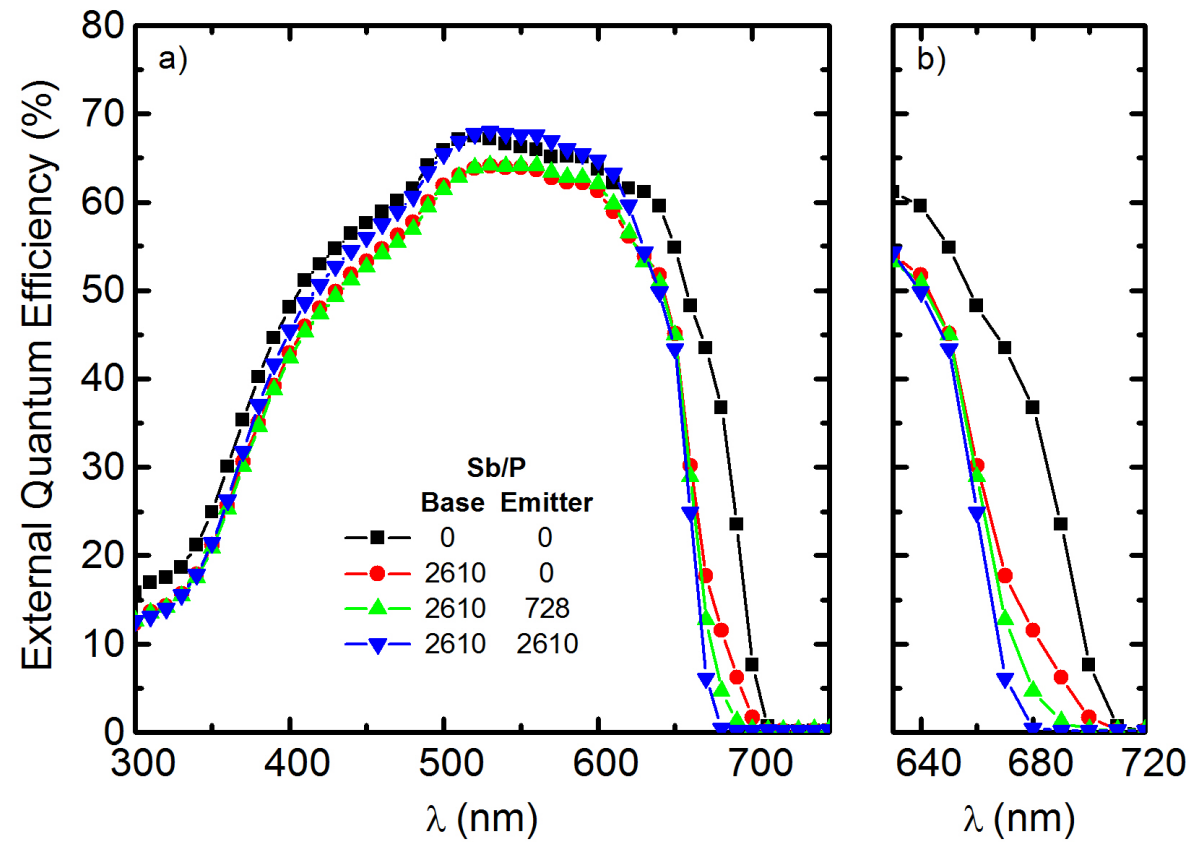

Figure 4: (a) EQE of GaInP solar cells grown without Sb flow (black squares) and with an $\mathrm{Sb} / \mathrm{P}$ ratio of $2610 \mathrm{ppm}$ in the base layer together with 0, 728 and $2610 \mathrm{ppm}$ in the emitter layer (red circles, green triangles and blue triangles, respectively). (b) Zoom in of the cut-off wavelength region of plot (a). 

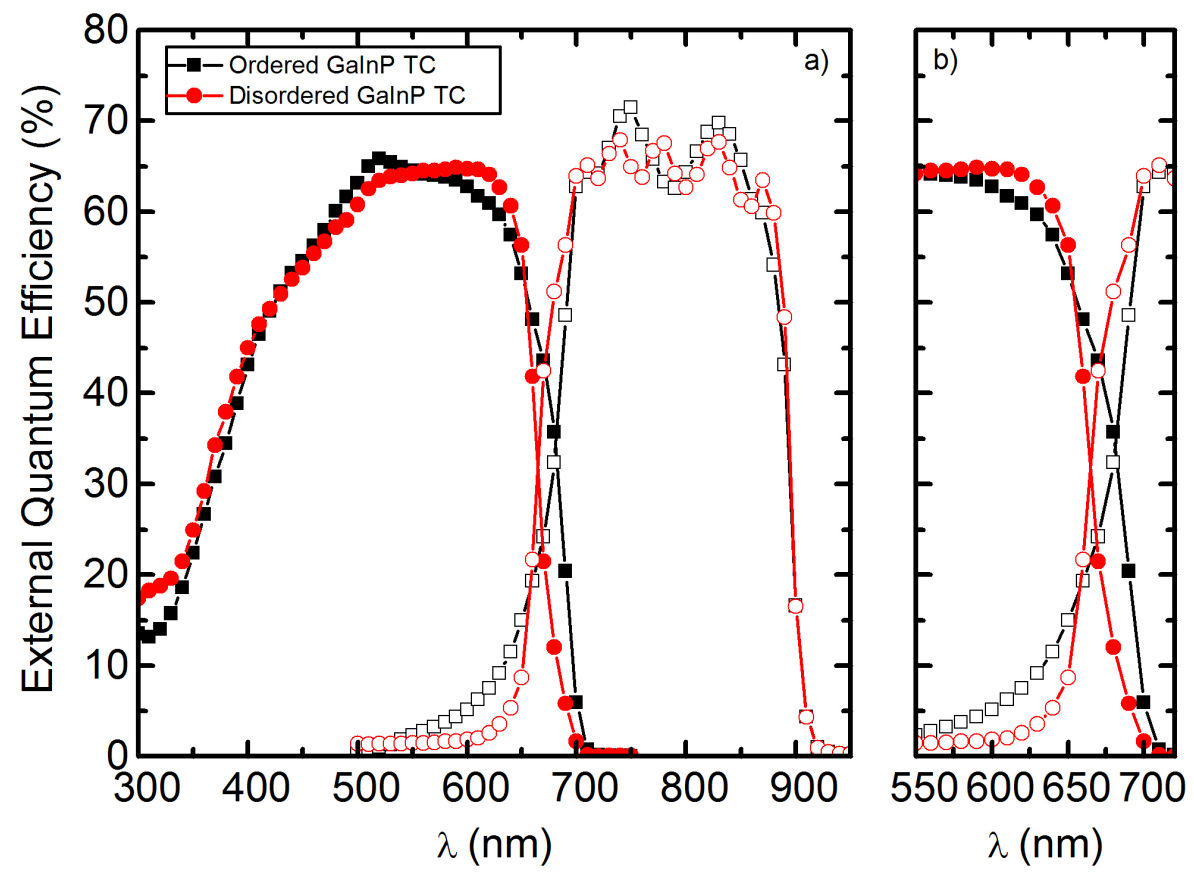

Figure 5: (a) EQE of GaInP and $\mathrm{Ga}(\mathrm{In}) \mathrm{As}$ subcells of a triple junction solar cell. Red circles correspond to the cell using $\mathrm{Sb}$ in the GaInP; whilst black squares correspond to the reference growth with no Sb. (b) Zoom of the region of interest in (a), where the differences in the EQE due to the effect of Sb can be observed 


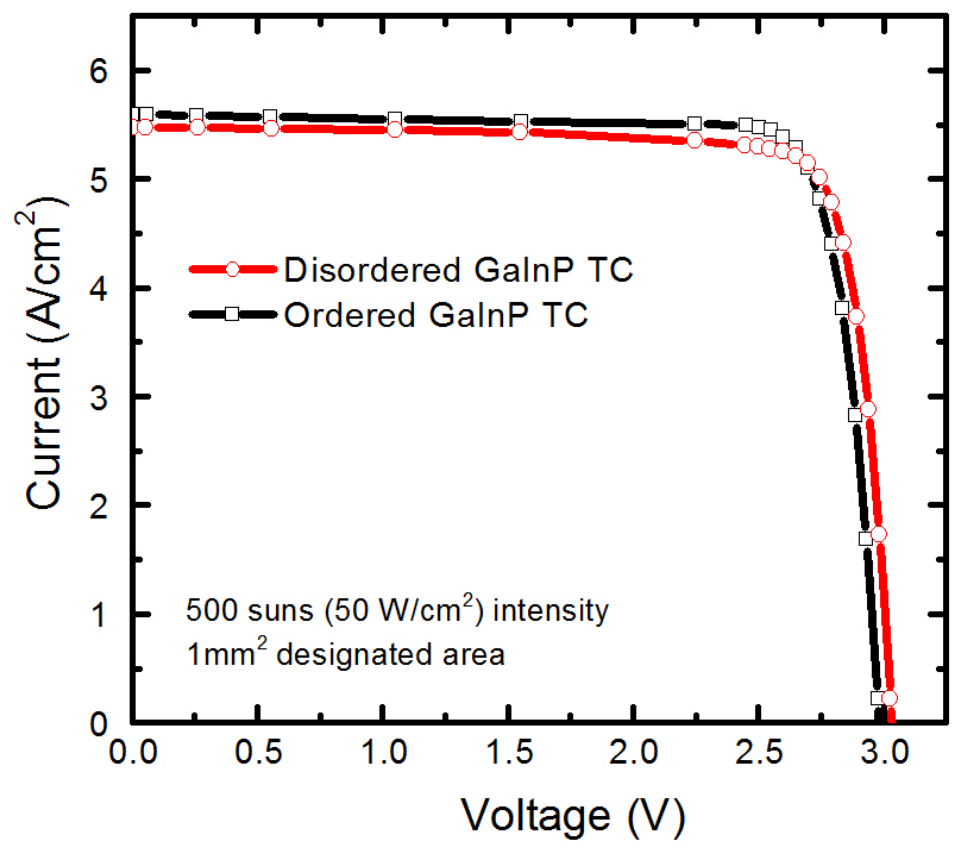

Figure 6: I-V curves of the triple-junction solar cells of Fig.5 at 500 suns. Black squares and red circles represent the IV curve of the devices with an ordered and disordered GaInP top cell, respectively. 